\title{
A velocidade de alcance durante tarefas de atenção e memória - um indicador de fragilidade em idosos
}

\author{
Daniana Michelle, ${ }^{1}$ Miguel Pires, ${ }^{1}$ Marlene Rosa ${ }^{1,2}$
}

\begin{abstract}
Resumo
Introdução: Diversas ferramentas de avaliação multidimensionais foram desenvolvidas para medir a fragilidade; no entanto, a sua implementação é complexa e a grande maioria está focada na marcha. Isto tem dificultado a avaliação e a previsão da fragilidade em idosos sem preservação da marcha. Objetivo: Caracterizar a velocidade de alcance durante atividades de atenção e memória, em população geriátrica em diferentes estados de fragilidade; e caracterizar os fatores que mais contribuíram para o fenótipo da fragilidade. Metodologia: Foi conduzido estudo descritivo exploratório, com idosos institucionalizados em 4 residências da região centro de Portugal, numa amostra de pessoas com idade $\geq 60$ anos, que compreendessem 2 comandos verbais e não acamados. Os participantes foram estratificados de acordo com os perfis categóricos de fragilidade, baseados em um conjunto de 9 variáveis: idade; relações sociais; sono; comorbidades; função motora de membro superior; função cognitiva; depressão; atividades de vida diária; e atividades instrumentais de vida diária. Foram comparadas as velocidades nos testes de alcance entre os indivíduos nas diferentes categorias de fragilidade, utilizando o teste de kruskal-wallis ( $\mathrm{p}<0,05)$. Resultados: Foram avaliadas 104 pessoas idosas: 51 pessoas foram classificadas como Robustas (G1), 36 Pré-frágeis (G2) e 17 Frágeis (G3). A velocidade de alcance em tarefas de atenção foi bom preditor para a distinção dos perfis categóricos de fragilidade G1-G3 ( $\mathrm{p}=0,000$ ) e $\mathrm{G} 2-\mathrm{G} 3$ ( $\mathrm{p}=0,004)$; o alcance em tarefas de memória fez distinção entre o G1-G3 ( $\mathrm{p}=0,028$ ). Conclusão: A velocidade de alcance em tarefas de atenção e memória demonstrou ser um indicador promissor para discriminação dos perfis de fragilidade em idosos institucionalizados.
\end{abstract}

Descritores: Velocidade de alcance; Fragilidade; Idosos.

\section{Abstract \\ Reaching velocity during attention and memory tasks - An indicator of frailty in elderly}

Introduction: Several multidimensional assessment tools have been developed to measure frailty, but their implementation is complex and the vast majority are focused on gait. This has hindered the assessment and prediction of frailty in the elderly without gait preservation. Objective: To characterize processing speed during attention and memory tasks in the geriatric population in different states of frailty; to characterize the factors that most contribute to the frailty phenotype. Methodology: An exploratory descriptive study
1. Escola Superior de Saúde, Instituto Politécnico de Leiria.

2. Center for Innovative Care and Health Technology (ciTechCare).

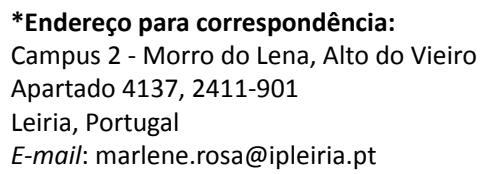

was conducted in 4 residential facilities in the central region of Portugal including institutionalized elderly aged $\geq 60$ years, using 2 verbal and non-verbal commands. Participants were stratified according to categorical profiles of frailty based on a set of 9 variables: age, social relations, sleep, comorbidities, upper limb motor function, cognitive function, depression, instrumental and basic daily activities. The processing speed tests compared the individuals in the different frailty categories, using the Kruskal-Wallis test $(\mathrm{p}<0.05)$. Result: One hundred and four elderly people were evaluated: 51 individuals were classified as Robust (G1); 36 as Pre-frail (G2); and 17 as Frail (G3). The processing speed in Attention Tasks was a good predictor for the categorization of frailty profiles G1-G3 $(p=0.000)$ and G2-G3 ( $p=0.004)$; the range of processing speed in Memory Tasks made a distinction between G1-G3 ( $\mathrm{p}=0.028$ ). Conclusion: Processing speed in attention and memory tasks has been shown to be a promising indicator for the characterization of frailty profiles in elderly people who are institutionalized.

Keywords: Processing speed; Frailty; Elderly.

\section{Resumen}

Velocidad en el alcance durante las tareas de atención y memoria - un indicador de fragilidad en los ancianos

Introducción: Diversas herramientas de evaluación multidimensionales fueron desarrolladas para medir la fragilidad, sin embargo su implementación es compleja y la gran mayoría están enfocadas en la marcha. Esto ha dificultado la evaluación y previsión de la fragilidad en ancianos sin preservación de la marcha. Objetivo: Caracterizar la velocidad del alcance durante 


\section{Artigo original}

actividades de atención y memoria, en población geriátrica; caracterizar los factores que más contribuyeron al fenotipo de la fragilidad. Métodos: Se realizó un estudio exploratorio descriptivo con los ancianos asilados en 4 hogares en centro de Portugal en una muestra de personas con edades $\geq 60$ años que entienden 2 comandos verbales. Los participantes fueron estratificados de acuerdo con los perfiles catégoricos de fragilidad basados en un conjunto de 9 variables: edad, relaciones sociales, sueño, comorbilidades, función motora de miembro superior, función cognitiva, depresión, avd's, aivd's. Se compararon las velocidades en las pruebas de alcance entre los ancianos en las diferentes categorías de fragilidad, utilizando la prueba de kruskal-wallis ( $\mathrm{p}<0,05)$. Resultado: Se evaluaron 104 personas: 51 personas se clasificaron como Robustas (G1), 36 Pre-frágiles (G2) y 17 Frágeis (G3). La velocidad del alcance en Tareas de Atención fue buen predictor para la distinción de los perfiles categóricos de fragilidad G1-G3 ( $p=0,000$ ) y G2-G3 ( $p=0,004$ ); el alcance en Tareas de Memoria hizo distinción entre el G1-G3 ( $p=0,028)$. Conclusión: La velocidad del alcance en tareas de atención y memoria demostró ser un indicador prometedor para dicriminación de los perfiles de fragilidad en ancianos institucionalizados.

Palabras clave: Velocidad de alcance; Fragilidad; Ancianos.

\section{Introdução}

O envelhecimento populacional é uma realidade no mundo, com implicações para a pessoa idosa, para as famílias e para as políticas sociais e de saúde. O número de pessoas idosas deverá aumentar drasticamente, em nível mundial, nas próximas décadas, e, em 2050, espera-se que o número de pessoas com 60 anos aumente para 2,1 bilhões e para 3,2 bilhões em 2100. ${ }^{1}$ Em Portugal o número de pessoas com mais de 65 anos poderá passar de 2,1 para 2,8 milhões de pessoas, com um aumento dos valores de índice de envelhecimento, passando de 147 para 317 idosos, por cada 100 jovens, entre 2015 e $2080 .{ }^{2} \mathrm{O}$ cenário do envelhecimento populacional agrava-se com a realidade de que, aproximadamente, $10 \%$ das pessoas com mais de 65 anos e $25 \%$ a $50 \%$ das pessoas com mais de 85 anos apresentam-se com estados frágeis de saúde. ${ }^{3}$ Por esta razão, o conceito de fragilidade tem recebido atenção especial nos últimos anos; e várias definições de fragilidade têm sido propostas. ${ }^{4}$

A fragilidade é uma síndrome do envelhecimento, que resulta de um declínio gradativo, em múltiplos sistemas orgânicos. ${ }^{5}$ Como a fragilidade conduz a uma vulnerabilidade e a uma redução da capacidade de manter a homeostase, os idosos estão predispostos a déficits funcionais, comorbidades e maior índice de mortalidade. ${ }^{6}$ Pelo impacto da fragilidade na saúde geral do idoso, é importante detectar e prevenir o estado de fragilidade, implementando linhas estratégicas para a prestação de cuidados adaptados aos idosos frágeis ou em risco de fragilidade. ${ }^{7}$

Embora numerosas ferramentas de avaliação tenham sido desenvolvidas para medir a fragilidade, os instrumentos frequentemente usados envolvem a colheita de múltiplos fatores, desde cognição, função geral de saúde, independência funcional, suporte social, medicação, estado nutricional, entre outros. ${ }^{8}$

Apesar de serem instrumentos válidos e fiáveis, a sua implementação implica uma colheita de informação bastante demorada, o que desencoraja a sua utilização em realidades institucionais em que os recursos humanos podem não ter ao seu dispor o tempo ou as condições necessárias. ${ }^{9}$

Como alternativa aos índices de fragilidade, alguns autores têm explorado a utilização de testes funcionais, por se tratarem de alternativas mais rápidas e igualmente válidas para avaliar e descriminar graus/ níveis de fragilidade nos idosos. Neste contexto, os testes de avaliação da velocidade da marcha têm sido altamente recomendados ${ }^{1011}$

Contudo, a marcha está muitas vezes debilitada na população geriátrica, ${ }^{12}$ caracterizando-se por afetar cerca de $60 \%$ dos idosos com mais de 80 anos o que dificulta a implementação destes testes numa grande percentagem da população geriátrica.

Considerando a percentagem significativa de idosos com disfunções na marcha, a avaliação de um índice de fragilidade baseado na função dos membros superiores pode ser de grande utilidade num contexto geriátrico, tal como concluem os autores Toosizadeh et al. ${ }^{13}$ Este é um dos primeiros autores a validar um índice de fragilidade baseado na rapidez do movimento de flexão-extensão do cotovelo, medido através de sensores de movimento. ${ }^{13}$ No entanto, a implementação de testes que dependem do uso de tecnologias envolve custos e análise complexa de dados, dificultando a sua implementação em contexto clínico ou institucional. Por essa razão, os autores deste artigo desenvolveram um método simples, rápido e fácil de implementar, e que reporta a velocidade de alcance do membro superior como um potencial indicador de fragilidade.

Assim, este estudo teve como objetivo caracterizar a velocidade de alcance durante atividades de atenção 
e memória, em população geriátrica estratificada em estados Frágil, Pré-frágil e Robusto. Comprovando-se a velocidade de alcance como indicador de fragilidade nos idosos, os seus valores serão diferentes nos grupos com diferentes níveis de fragilidade.

\section{Métodos e materiais}

\section{Tipo de estudo e aprovação ética}

Trata-se de um estudo descritivo exploratório, que foi realizado em quatro residências da região Centro de Portugal. O estudo recebeu aprovação do Comitê de Ética de cada instituição. Os participantes potencialmente elegíveis foram identificados e abordados pelo diretor-técnico de cada instituição. Aqueles que expressaram interesse foram contatados pelo pesquisador para agendamento de uma primeira reunião. Durante essa reunião foi providenciada informação escrita detalhada sobre o estudo aos participantes e esclarecida qualquer dúvida sobre os procedimentos do estudo. Compreendida toda a informação, foi pedida a assinatura do termo de consentimento livre e esclarecido.

\section{Seleção da amostra}

Foram incluídos participantes com: (i) idade igual ou superior a 60 anos, (ii) não acamados e (iii) capazes de compreender, pelo menos, dois comandos verbais simples. Foram excluídos idosos acamados e em estados graves de alterações de consciência ou com afasia global ou de compreensão. A seleção da amostra e a colheita dos dados ocorreu no período de março a abril de 2019.

\section{Instrumentos de avaliação}

\section{Cálculo do Índice de Fragilidade}

A avaliação foi realizada em ambiente reservado,com duração média de 60 a 90 minutos, por dois pesquisadores treinados. Para o cálculo do Índice de Fragilidade foi recolhida informação sociodemográfica e clínica através de um questionário e instrumentos de avaliação individuais. Estes dados recolhidos foram utilizados para a estratificação em níveis de fragilidade, segundo o método descrito por Jones et al.(14) Assim, a estratificação resultou da consideração das seguintes 9 variáveis:

FÓRMULA FRAGILIDADE $=$ [IDADE $]+$ [RELAÇÕES SOCIAIS $]$ $+[$ SONO $]+[$ COMORBIDADES $]+[$ FUNÇÃO MOTORA DE MEMBRO SUPERIOR] + [FUNÇÃO COGNITIVA] + $[$ DEPRESSÃO $]+[$ AVD's $]+[$ AIVD's $]$
Com base nesta fórmula, gerou-se um fenótipo de fragilidade considerando as diferentes percentagens de critérios afetados, a referir:

(A) Idosos Robustos - apresentaram até ao máximo de $25 \%$ de critérios afetados (0-3 fatores de risco);

(B) Idosos em estado Pré-frágil - apresentaram entre 44\%-55\% de critérios afetados ( 4 e 5 fatores de risco);

(C) Idosos em estado Frágil - apresentaram entre $60 \%-100 \%$ de critérios afetados (6-9 fatores de risco);

Para a classificação de cada critério do Índice de Fragilidade como estando ou não afetado, foram considerados os seguintes parâmetros:

[IDADE] - As pessoas que tinham idade igual ou superior a 80 anos eram classificadas com indicador positivo para fragilidade. ${ }^{4}$

[RELAÇÕES SOCIAIS] - A caracterização deste critério baseou-se nos seguintes parâmetros: (i) estado civil (solteiro, casado, viúvo e divorciado); (ii) se tinham filhos ou netos; (iii) se coabitavam com alguém; (iv) se mantinham contato com a família (sim ou não); (v) o tipo de contato (pessoal, por telefone, internet ou por carta); (vi) tipo de apoio institucional (convívio, centro de dia, reabilitação física ou institucionalizado). ${ }^{15}$ Para este critério, foram considerados indicadores positivos para fragilidade as seguintes condições: (1) pessoas solteiras, sem filhos e institucionalizadas; e (2) viúvos, institucionalizados que contatavam a família apenas por telefone ou não contatavam de nenhuma forma.

[SONO] - Foi avaliado, no seu aspecto geral e em seus possíveis transtornos, através dos seguintes parâmetros: (i) auto-relato do número de horas de sono ininterruptas durante a noite; (ii) se apresentavam algum problema de sono atualmente (sim ou não); (iii) se sim (quais os problemas, quais estratégias de solução, e se utilizam alguma medicação para dormir). ${ }^{16}$ Foi considerado indicador positivo para fragilidade: (1) as pessoas que relataram dormir menos de 4 horas ininterruptas por noite; e ou (2) que dormiam de 4 a 6 horas, associado ao autorrelato de problemas de sono.

[COMORBIDADES] - A presença de comorbidades foi verificada através do (i) número de doenças diagnosticadas e de sua tipologia. Foi considerado ${ }^{17}$ indicador positivo para fragilidade, as pessoas que apresentaram três ou mais diagnósticos.

[FUNÇÃO MOTORA DO MEMBROSUPERIOR] - A função motora de membro superior foi avaliada através de dois instrumentos: a força de preensão manual e o controle sensório-motor do membro superior. Considerando 


\section{Artigo original}

estes 2 instrumentos, foi assumido como critério de fragilidade: 1) alteração de força de preensão manual nos dois membros através da dinanometria; e ou 2) pessoas que apresentavam alteração de força de preensão manual em apenas um membro e comprometimento severo ou total na escala de avaliação de função do membro superior Fulg Meyer (FM). A força de preensão manual foi aferida com dinamómetro k-force, em ambas as mãos. Para a medição deste teste, o idoso permaneceu sentado, com os pés apoiados no solo e o membro superior em teste com ombro em adução, cotovelo $90^{\circ}$ de flexão e antebraço na posição neutra. Os participantes realizaram três preensões intercaladas e foi selecionada a melhor tentativa. Depois do ajuste para gênero e idade, os valores que estivessem abaixo do normal foram considerados alterados. ${ }^{18} \mathrm{O}$ controle sensório-motor foi avaliado através da (FM), na sua versão para o membro superior, que tem classificação máxima igual a 100 pontos, a qual define função motora normal. Tendo em consideração o seu score máximo, foram estabelecidas ainda quatro classes de comprometimento motor: (i) comprometimento motor severo (pontuação<50) - significa pequena ou nenhuma movimentação voluntária dos membros afetados (ii) comprometimento motor grave (pontuação 51-84); (iii) comprometimento motor moderado (pontuação 8595) - existe movimento voluntário, contudo a função da mão pode estar altamente comprometida e (iv) comprometimento motor leve (pontuação 96-99). ${ }^{19}$

[FUNÇÃO COGNITIVA] - Foi implementado o mini exame do estado mental (MEEM), um breve questionário constituído de 2 partes: (i) uma que abrange orientação, memória e atenção, com pontuação máxima de 21 pontos; (ii) e outra que aborda habilidades específicas como nomear e compreender, com pontuação máxima de 9 pontos, totalizando um score de 30 pontos. Qualquer pontuação igual ou superior a 27 (de um total de 30 pontos) é efetivamente normal. Abaixo disso, a pontuação pode indicar perda cognitiva grave ( $\leq 9$ pontos), moderada (10 a 20 pontos) ou leve ( 21 a 24 pontos). ${ }^{20}$ No entanto, esta pontuação deve ser corrigida de acordo com a escolaridade e a idade. Em pessoas com (i) mais de 11 anos de escolaridade, considera-se a função cognitiva afetada quando a pontuação é $\leq 27$; (ii) numa pessoa com 1 a 11 anos de escolaridade, considera-se que a cognição está afetada quando $\leq 22$; e (iii) numa pessoa sem escolaridade, a cognição está afetada se a pontuação for $\leq 9 .{ }^{21}$ No presente estudo, a pontuação foi ajustada à escolaridade dos participantes e, de acordo com esse critério, sempre que os participantes apresentavam perda cognitiva, eram classificados com indicador positivo para fragilidade.

[DEPRESSÃO] - A Escala de Depressão Geriátrica (GDS) foi utilizada para o rastreio da depressão e avalia aspectos cognitivos e comportamentais tipicamente afetados na população idosa. A informação é obtida através de questionário direto, com 30 questões de resposta dicotômica (sim ou não). As respostas sugestivas de existência de depressão correspondem a 1 ponto. Num score total máximo de 30 pontos, (i) os valores de 0-10 são indicadores de ausência de depressão; (ii) 11-20 de depressão ligeira; e (iii) de 21 a 30, são indicadores de depressãograve. ${ }^{22}$ As pessoas que apresentaram pontuação acima de 20 pontos eram classificadas com indicador positivo para fragilidade.

[AVD'S] O Índice de Barthel (ADL) ${ }^{23}$ é um instrumento que avalia o nível de independência em 10 atividades básicas de vida diária: alimentação, higiene pessoal, uso de toalete, tomar banho, vestir e despir, continência urinária, controle de esfíncteres, deambular, transferência da cadeira para a cama, subir e descer escadas. Foi aplicada a versão original com uma pontuação que varia de 0-100. Assim, (i) pontuações de 90-100 indicam independência funcional; (ii) 60-89, leve dependência funcional; (iii) 40-59, moderada dependência funcional; (iv) 20-39, severa dependência funcional; (v) 0-19, total dependência funcional. ${ }^{24-23}$ As pessoas foram classificadas com indicador positivo para fragilidade, se total ou severamente dependentes, com pontuação inferior a 39 pontos.

[AIVD’S] Através do Índice Lawton (IADL), ${ }^{25}$ foi avaliado o nível de dependência para realização de 8 tarefas instrumentais de vida diária, nomeadamente: a utilização do telefone, realização de compras, preparação das refeições, tarefas domésticas, lavagem da roupa, utilização de meios de transporte, gestão da medicação e responsabilidade com assuntos financeiros. ${ }^{26} \mathrm{~A}$ pontuação final varia entre 0 a 8 pontos, e entre $0-5$ pontos para homens, e não contabiliza a preparação das refeições, as tarefas domésticas e a lavagem da roupa. Para cada tarefa, a pontuação varia entre dependente ( 0 pontos) ou independente (1 ponto), e as classificações totais foram classificadas como independente (pontuação=8 para mulheres versus pontuação $=5$ para homens), dependência moderada (pontuação=4 versus pontuação=3) e dependência grave (pontuação=0). No presente estudo, os pontos foram ajustados ao gênero, e as pessoas com dependência grave foram classificadas com indicador positivo para fragilidade.

\section{Protocolo de Avaliação da Velocidade de Alcance durantes tarefas de atenção e durante tarefas de memória}

O projeto Agilidades dispõe do teste de alcance para tarefas de atenção (AA) e alcance para tarefas de memória (AM), ambos desenvolvidos no Instituto Politécnico de Leiria. ${ }^{27}$ A realização destes testes requer um conjunto sistematizado de movimentos, especialmente adaptados às possibilidades e às necessidades das pessoas idosas. $\mathrm{O}$ instrumento de avaliação consiste num tabuleiro com 24 figuras 
geométricas, com formas e cores diferentes, cada uma com $12 \mathrm{~cm}$ por $14 \mathrm{~cm}$ de dimensão, distribuídas em quatro linhas e seis colunas, dispostas lado a lado. A disposição destas figuras é separada por uma linha média que dispõe de forma simétrica, com três colunas para o lado direito e três para o esquerdo. Entre a $3^{\circ}$ e a $4^{\circ}$ linha e a $5^{\circ}$ e $6^{\circ}$ coluna, foram colocadas mais duas figuras do lado direito e duas do lado esquerdo, contabilizando um total de 28 figuras. As figuras foram distribuídas de forma que a distância da base de início do jogo a cada uma das figuras pudesse ser aferida (Figura 1).

Este tabuleiro, desenhado para o Teste do Alcance, tem a flexibilidade de facultar dois testes de tarefas diferentes, nomeadamente as Tarefas de Atenção e de Memória:

(A) Teste do Alcance com Tarefa de Atenção consta de 10 cartas, de figuras geométricas com formas e cores diferentes. Cada carta tem uma informação adicional que sugere qual o membro que deverá realizar o alcance, podendo constar a opção de alcançar a figura com a mão direita ou com a mão esquerda. A Figura 2 apresenta uma carta com indicações para o Alcance com Tarefa de Atenção. A distância final das figuras alcançadas neste jogo foi de 8.9 metros.

(B) Teste do Alcance com Tarefa de Memória utiliza o mesmo tabuleiro, mas com cartas que apresentam desafios diferentes. Este teste é constituído por 9 cartas, formando o conjunto de três pares de figuras. Depois de alcançado cada par de cartas, é solicitado ao idoso que repita o alcance realizado nas duas últimas cartas através do processo de memorização e retenção motora e visual. A contabilização total das 9 cartas, mais as suas repetições, resulta numa distância total de 12.4 metros. A Figura 3 apresenta a carta com a instrução que incentiva o idoso a repetir o alcance do último par de figuras em jogo, apenas recorrendo à sua memória de trabalho.

Para a realização do teste, o tabuleiro é colocado numa mesa de altura ajustada a cada participante, a uma distância de $5 \mathrm{~cm}$ da face anterior do tronco do examinado. Esse espaço foi necessário para que cada participante pudesse colocar a mão direita e a esquerda sobre a mesa, em dois locais indicados na base do tabuleiro. O teste somente tem início quando os participantes estão sentados, com as mãos
Figura 1. Tabuleiro com as figuras para desempenho do teste do alcance com tarefa de atenção e do teste do alcance com tarefa de memória

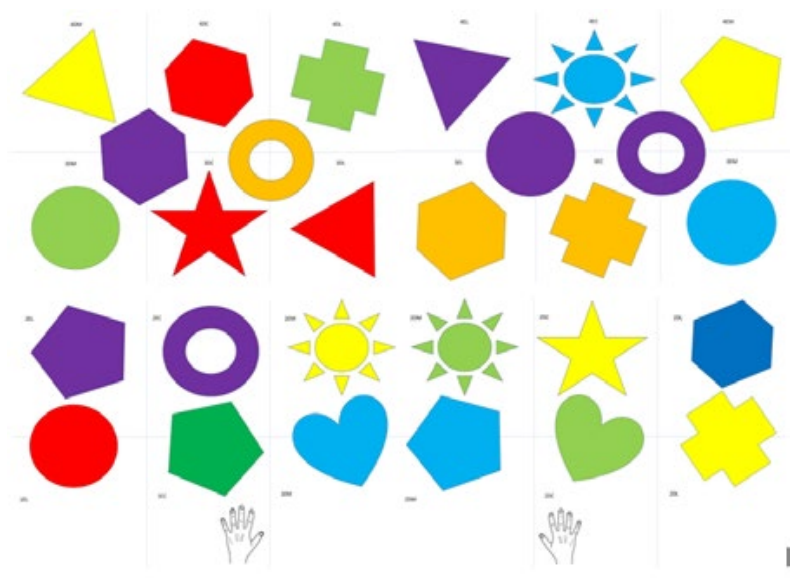

Figura 2. Exemplo de uma carta para execução de alcance numa tarefa de atenção em que o participante deve encontrar a figura representada, dentre todas as figuras no tabuleiro, alcançando-a rapidamente com a mão direita (conforme indicado pela seta)

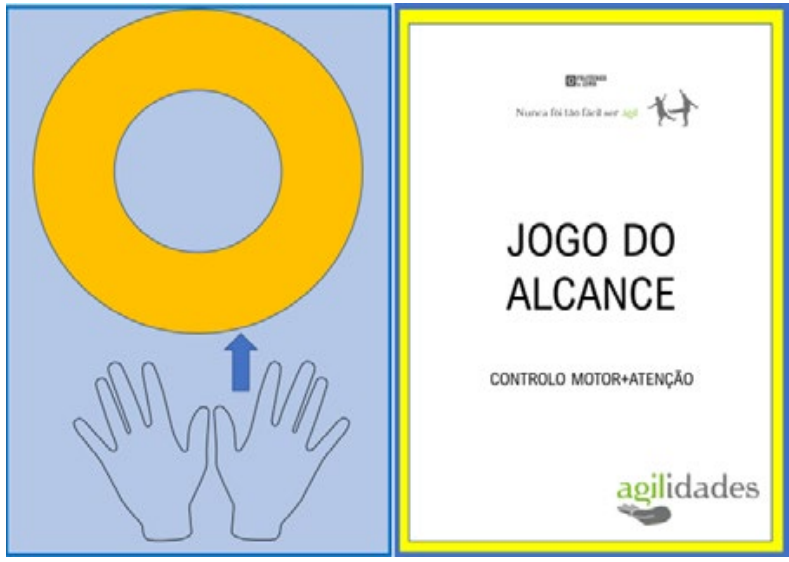

Figura 3. Exemplo de uma carta para repetição do alcance do último par de figuras em jogo

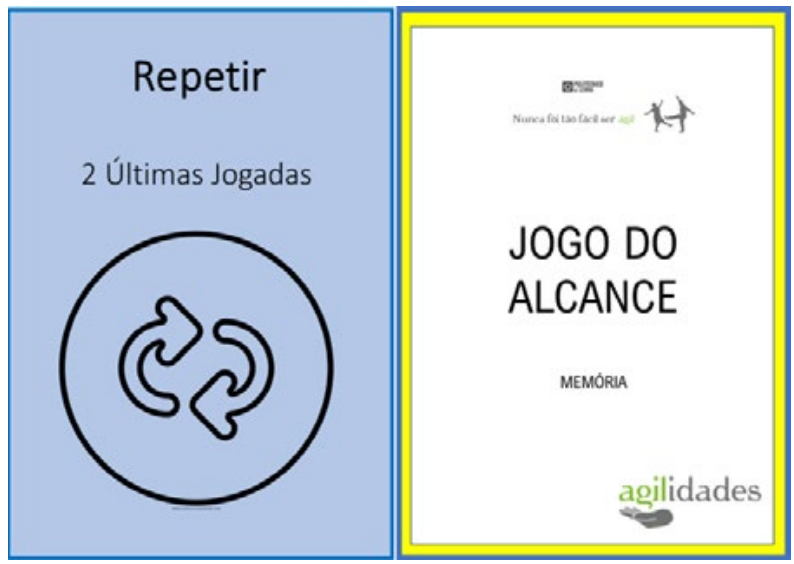




\section{Artigo original}

posicionadas adequadamente e com o tronco em posição central ao tabuleiro. As instruções a respeito do objetivo do teste são dadas a cada participante e, só após o entendimento das regras, o examinador inicia cronometragem do tempo, mostrando imediatamente a primeira carta com a figura a ser alcançada. Os instrumentos de avaliação AA e AM foram aplicados em cada participante, o tempo total do teste foi cronometrado e a distância percorrida (previamente conhecida) foi usada para cálculo da velocidade, em metros por segundo (m/s). As pessoas que apresentaram 5 ou mais erros durante a aplicação dos testes foram consideradas sem critério para realização, e a sua performance foi considerada nula.

\section{Análise estatística}

Com base nos dados do Índice de Fragilidade, os participantes foram estratificados em 3 grupos: o grupo de pessoas Frágeis; o grupo de pessoas em estado Pré-frágil; e o grupo de pessoas em estado Robusto. Estatística descritiva (média, desvio padrão e frequências) foi usada para descrever as características sociodemográficas, clínicas e funcionais de cada grupo de fragilidade e para caracterizar a relevância de cada uma destas características para o diagnóstico de fragilidade. A estatística não paramétrica (teste de Kruskal-Wallis) foi aplicada para comparar os valores de velocidade de alcance nas amostras dos três grupos (Robusto, Pré-frágil e Frágil). Foi considerado um p <0,05 como significante.

\section{Resultados}

\section{Estratificação dos idosos em estados de fragilidade}

A amostra do presente estudo apresentou um total de 104 participantes, com média de idade e desvio padrão de 78.00 \pm 10.27 anos. De acordo com a fórmula do índice de fragilidade, os participantes foram distribuídos em três categorias diferentes: 51 idosos (46.7\%) receberam classificação de Robustos, $36(37,1 \%)$ receberam a classificação de Pré-frágeis e 17 (16,2\%) foram classificados como idosos Frágeis. De acordo com os domínios da fórmula para cálculo da fragilidade (Relações Sociais, Sono, Comorbidades, Função Motora de Membro Superior, Função Cognitiva, Depressão, AVD'S e AIVD'S), os respectivos número e percentagem de pessoas que apresentaram indicador positivo para esses parâmetros estão demonstrados na Tabela 1 .
De todas as características avaliadas e apresentadas na Tabela 1, as que mais contribuíram de forma individual para a estratificação dos participantes nos perfis de fragilidade foram: (i) a idade, que esteve presente em 56,73\% da amostra total e em 82,35\% das pessoas do grupo Frágil; (ii) a força de preensão manual nos dois membros, que se apresentou alterada em $59.62 \%$ da amostra total, em $80.56 \%$ das pessoas do grupo Pré-frágil e em 82,35\% do grupo Frágil; (iii) o deficit cognitivo, que esteve presente em 51,92\% da amostra total, em 63,89\% do grupo Pré-frágil e em 82,35\% do grupo Frágil; (iv) a dependência nas Atividade de Vida Diária (ADL), que esteve presente em 47,12\% da amostra total e em 100\% do grupo Frágil; (v) a dependência nas Atividades Instrumentais de Vida Diária, que esteve presente em $71 \%$ da amostra total, em 50,98\% da pessoas do grupo Robusto, em 91,67\% do grupo Pré-frágil e em 88,24\% do grupo Frágil.

\section{Valores de velocidade de alcance nos Grupo de Idosos em estado Frágil, Pré-Frágil e Robusto}

A média das velocidades (m/s) nos testes AA e AM demonstrou valores estatisticamente diferentes entre, pelo menos, um par de grupos em estudo (Robusto, Pré-frágil e Frágil), como mostra a Tabela 2 e o Gráfico 1 (A-B). Especificamente para a tarefa de atenção, o grupo Robusto apresentou uma média de velocidade superior $(\mathrm{G} 1=0,080 \mathrm{~m} / \mathrm{s})$ à média apresentada pelo grupo Pré-frágil (G2=0,060m/s), seguido do grupo Frágil (G3=0,026m/s). Assim, a velocidade demonstrou ser estatisticamente diferente entre o G1 e G3 (p=0,000) e entre o G2 e G3 ( $p=0,004$ ), conforme demostrado no Gráfico 1A. Para a tarefa de memória, o grupo Robusto apresentou uma média de velocidade superior (G1=0,093m/s) à média apresentada pelo grupo Pré-frágil (G2=0,053m/s), seguido do grupo Frágil $(\mathrm{G} 3=0,038 \mathrm{~m} / \mathrm{s})$. Ao comparar os grupos, a diferença foi estatisticamente significativa entre o G1 e G3 $(\mathrm{p}=0,028)$, conforme demostrado no Gráfico $1 \mathrm{~B}$.

Ao analisar a curva de percentis para o alcance em tarefa de atenção, identificou-se que existe $10 \%$ da população em estudo, na qual não foi possível diferenciar o critério de velocidade de alcance, de acordo com os perfis de fragilidade. A partir do percentil 25, os grupos Frágil e Robusto destacam-se nos seus valores de velocidade de alcance, mantendose afastados nos restantes percentis. Assim, a população Robusta no percentil 50-75\% apresentou valores de velocidade entre os $0,073 \mathrm{~m} / \mathrm{s}$ e os $0,092 \mathrm{~m} / \mathrm{s}$, enquanto que a população Frágil no percentil 50-75\% apresentou 
Tabela 1. Distribuição das características sociodemográficas, clínicas e funcionais da amostra e descrição, em cada grupo, de acordo com o seu Índice de Fragilidade (Robusto, Pré-frágil e Frágil). Deficit de saúde que contribuíram como fator de risco para estratificação da fragilidade

\begin{tabular}{|c|c|c|c|c|c|}
\hline & & $\begin{array}{c}\text { Robusto } \\
N=51\end{array}$ & $\begin{array}{l}\text { Pré-Frágil } \\
\mathrm{N}=36\end{array}$ & $\begin{array}{l}\text { Frágil } \\
\mathrm{N}=17\end{array}$ & $\begin{array}{c}\text { Total } \\
\mathrm{N}=104\end{array}$ \\
\hline Idade (anos) & média \pm desvio padrão & $77,23 \pm 10,58$ & $78,11 \pm 10,21$ & $84,33 \pm 6,19$ & $78 \pm 10,271$ \\
\hline Idade (anos) & $+80 \operatorname{anos}(\mathrm{N} ; \%)$ & $\begin{array}{c}25 \\
(49,01 \%)\end{array}$ & $\begin{array}{c}20 \\
(55,56 \%)\end{array}$ & $\begin{array}{c}14 \\
(82,35 \%)\end{array}$ & $\begin{array}{c}59 \\
(56,73 \%)\end{array}$ \\
\hline \multirow{2}{*}{ Relações sociais } & $\begin{array}{l}\text { Solteiro+sem filhos+ institucionalizados } \\
(\mathrm{N} ; \%) \text {; }\end{array}$ & $\begin{array}{c}4 \\
(7,84 \%)\end{array}$ & $\begin{array}{c}6 \\
(16,67 \%)\end{array}$ & $\begin{array}{c}1 \\
(5,88 \%)\end{array}$ & $\begin{array}{c}11 \\
(10,58 \%)\end{array}$ \\
\hline & $\begin{array}{l}\text { Viúvos+institucionalizados+sem } \\
\text { contacto com a família }(\mathrm{N} ; \%)\end{array}$ & $\begin{array}{c}4 \\
(7,84 \%)\end{array}$ & $\begin{array}{c}2 \\
(5,56 \%)\end{array}$ & $\begin{array}{c}2 \\
(11,76 \%)\end{array}$ & $\begin{array}{c}8 \\
(7,69 \%)\end{array}$ \\
\hline \multirow{2}{*}{ Sono (horas) } & $<$ de 4 horas por noite $(\mathrm{N} ; \%)$ & $\begin{array}{c}1 \\
(1,96 \%)\end{array}$ & $\begin{array}{c}2 \\
(5,56 \%)\end{array}$ & $\begin{array}{c}0 \\
(0 \%)\end{array}$ & $\begin{array}{c}3 \\
(2,88 \%)\end{array}$ \\
\hline & $\begin{array}{l}<4-6 \text { horas com relato de problemas } \\
\text { de sono. }(\mathrm{N} ; \%)\end{array}$ & $\begin{array}{c}2 \\
(3,92 \%)\end{array}$ & $\begin{array}{c}4 \\
(5,56 \%)\end{array}$ & $\begin{array}{c}3 \\
(17,65 \%)\end{array}$ & $\begin{array}{c}9 \\
(8,65 \%)\end{array}$ \\
\hline Comorbidades & $3 /+$ comorbidades $(\mathrm{N} ; \%)$ & $\begin{array}{c}2 \\
(3,92 \%)\end{array}$ & $\begin{array}{c}5 \\
(13,89 \%)\end{array}$ & $\begin{array}{c}3 \\
(17,65 \%)\end{array}$ & $\begin{array}{c}10 \\
(9,62 \%)\end{array}$ \\
\hline \multirow{2}{*}{$\begin{array}{l}\text { Força de } \\
\text { preensão } \\
\text { manual (KG/F) }\end{array}$} & Força alterada em ambos MS (N;\%) & $\begin{array}{c}19 \\
(37-25 \%)\end{array}$ & $\begin{array}{c}29 \\
(80,56 \%)\end{array}$ & $\begin{array}{c}14 \\
(82,35 \%)\end{array}$ & $\begin{array}{c}62 \\
(59,62 \%)\end{array}$ \\
\hline & $\begin{array}{l}\text { Força alterada no MSD/MDE + } \\
\text { Fugl-Meyer grave e severo (N;\%) }\end{array}$ & $\begin{array}{c}5 \\
(9,80 \%)\end{array}$ & $\begin{array}{c}1 \\
(2.78 \%)\end{array}$ & $\begin{array}{c}4 \\
(23.53 \%)\end{array}$ & $\begin{array}{c}10 \\
(9.62 \%)\end{array}$ \\
\hline Função cognitiva & Alteração cognitiva no MEEM & $\begin{array}{c}17 \\
(33.33 \%)\end{array}$ & $\begin{array}{c}23 \\
(63.89 \%)\end{array}$ & $\begin{array}{c}14 \\
(82.35 \%)\end{array}$ & $\begin{array}{c}54 \\
(51.92 \%)\end{array}$ \\
\hline Depressão & $\begin{array}{l}\text { Escala Geriátrica da Depressão } \\
\text { Score } \geq 20 \text { pontos }\end{array}$ & $\begin{array}{c}9 \\
(17.65 \%)\end{array}$ & $\begin{array}{c}14 \\
(38.89 \%)\end{array}$ & $\begin{array}{c}9 \\
(52.94 \%)\end{array}$ & $\begin{array}{c}32 \\
(30.77 \%)\end{array}$ \\
\hline AVD'S & $\begin{array}{l}\text { Índice de Barthel } \\
\text { Totalmente/severamente dependente }\end{array}$ & $\begin{array}{c}8 \\
(15.68 \%)\end{array}$ & $\begin{array}{c}23 \\
(63.89 \%)\end{array}$ & $\begin{array}{c}17 \\
(100 \%)\end{array}$ & $\begin{array}{c}49 \\
(47.12 \%)\end{array}$ \\
\hline AIVD'S & $\begin{array}{l}\text { Índice de Lawton } \\
\text { Dependência grave }\end{array}$ & $\begin{array}{c}26 \\
(50.98 \%)\end{array}$ & $\begin{array}{c}33 \\
(91.67 \%)\end{array}$ & $\begin{array}{c}15 \\
(88.24 \%)\end{array}$ & $\begin{array}{c}74 \\
(71.15 \%)\end{array}$ \\
\hline
\end{tabular}

Legenda: (KG/F) Quilograma força, MMSS Membros Superiores, MSD Membro Superior Direito, MSE Membro Superior Esquerdo, D direita, E esquerda, MEEM Mini Exame do Estado Mental, AVD'S Atividades de vida diária e AIVD'S Atividades instrumentais de vida diária.

Tabela 2. Comparação das velocidades $(\mathrm{m} / \mathrm{s})$ médias de alcance em tarefas de atenção e em tarefas de memória entre os perfis de fragilidade (Robusto, Frágil e Pré Frágil)

\begin{tabular}{|c|c|c|c|c|c|}
\hline & $\begin{array}{c}\text { Grupo } 1 \\
\text { Robusto } \\
\mathrm{N}=51\end{array}$ & $\begin{array}{c}\text { Grupo } 2 \\
\text { Pré-frágil } \\
\mathrm{N}=36\end{array}$ & $\begin{array}{c}\text { Grupo } 3 \\
\text { Frágil } \\
\mathrm{N}=17\end{array}$ & $\begin{array}{l}\text { Paired } \\
\text { p-valor }\end{array}$ & $\begin{array}{c}\text { G1-G2-G3 } \\
\text { (p-valor) }\end{array}$ \\
\hline Alcance na tarefa de atenção $(\mathrm{m} / \mathrm{s})$ & $\begin{array}{l}0,080 \mathrm{~m} / \mathrm{s} \\
\pm 0,065\end{array}$ & $\begin{array}{l}0,060 \mathrm{~m} / \mathrm{s} \\
\pm 0,031\end{array}$ & $\begin{array}{l}0,026 \mathrm{~m} / \mathrm{s} \\
\pm 0,026\end{array}$ & $\begin{array}{c}0,854^{\mathrm{G1}-\mathrm{G} 2} \\
0,000^{\mathrm{G1}-\mathrm{G} *} \\
0,004^{\mathrm{G} 2-\mathrm{G} 3 *}\end{array}$ & $0,000^{*}$ \\
\hline $\begin{array}{l}\text { Alcance funcional na tarefa de memória } \\
(\mathrm{m} / \mathrm{s})\end{array}$ & $\begin{array}{c}0,093 \mathrm{~m} / \mathrm{s} \\
\pm 0,080\end{array}$ & $\begin{array}{l}0,053 \mathrm{~m} / \mathrm{s} \\
\pm 0,051\end{array}$ & $\begin{array}{l}0,038 \mathrm{~m} / \mathrm{s} \\
\pm 0,051\end{array}$ & $\begin{array}{c}0,200^{\mathrm{G1}-\mathrm{G} 2} \\
0,028^{\mathrm{G} 1-\mathrm{G} 3} \\
0,792^{\mathrm{G} 2-\mathrm{G} 3}\end{array}$ & $0,020^{*}$ \\
\hline
\end{tabular}

Legenda: (G1) Robusto, (G2) Pré-frágil e (G3) Frágil; (m/s) metros por segundo, ( \pm ) desvio padão e * p-valor<0,05. 


\section{Artigo original}

Gráfico 1. Gráfico representativo das diferenças das velocidades médias no teste de alcance com tarefa de atenção (Gráfico 1.A), alcance com tarefa de memória (Gráfico 1.B) e os perfis da fragilidade

Alcance em Tarefas de Atenção

(A)

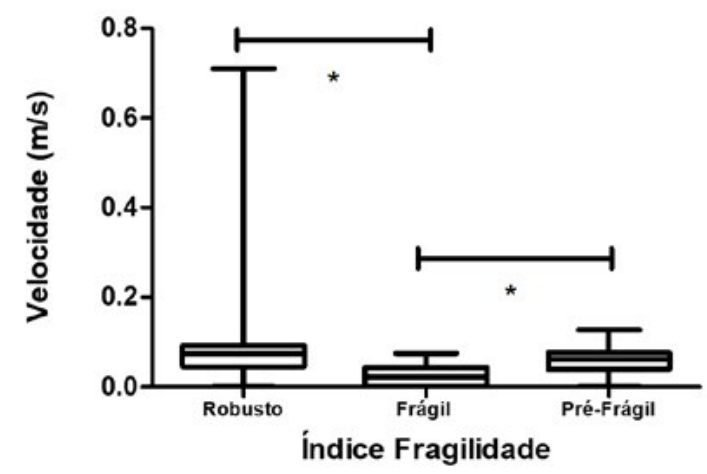

$*$ p-valor $<0,05$.

valores entre os $0,021 \mathrm{~m} / \mathrm{s}$ e $0,043 \mathrm{~m} / \mathrm{s}$, como disposto na Figura 1.

Ao analisar a curva de percentis para o alcance em tarefa de memória, identificou-se que existe $25 \%$ da população em estudo, na qual não foi possível diferenciar o critério de velocidade de alcance, de acordo com os perfis de fragilidade. A partir do percentil 25, o grupo Robusto destacou-se nos seus valores de velocidade de alcance, mantendo-se afastados nos restantes percentis. Assim, a população Robusta no percentil 50-95\% apresentou valores de velocidade ascendente entre os $0,088 \mathrm{~m} / \mathrm{s}$ e $0,233 \mathrm{~m} / \mathrm{s}$, enquanto que a população Pré-frágil no percentil 50$95 \%$ apresentou valores entre os $0,044 \mathrm{~m} / \mathrm{s} \mathrm{e} 0,151 \mathrm{~m} / \mathrm{s}$. O grupo dos idosos classificados em estado Frágil no percentil $50-90 \%$ apresentou valores entre os $0,006 \mathrm{~m} / \mathrm{s}$ e $0,116 \mathrm{~m} / \mathrm{s}$, como disposto na Figura 2.

\section{Discussão}

O presente estudo demonstrou que a velocidade média do alcance na tarefa de atenção e memória apresentou variabilidade significativa entre os perfis de fragilidade da população em estudo. Para distinção de pessoas que foram incluídas nos grupos Robusto, Pré-frágil e Frágil, o teste do alcance com tarefa de atenção demonstrou ser um indicador promissor, apresentando diferenças estatisticamente significativas entre as velocidades médias dos grupos Robusto e Frágil $(p=0,000)$ e entre as velocidades dos grupos Pré-frágil e Frágil $(\mathrm{p}=0,004)$. Adicionalmente, os valores de velocidade
Alcance em Tarefas de Memória

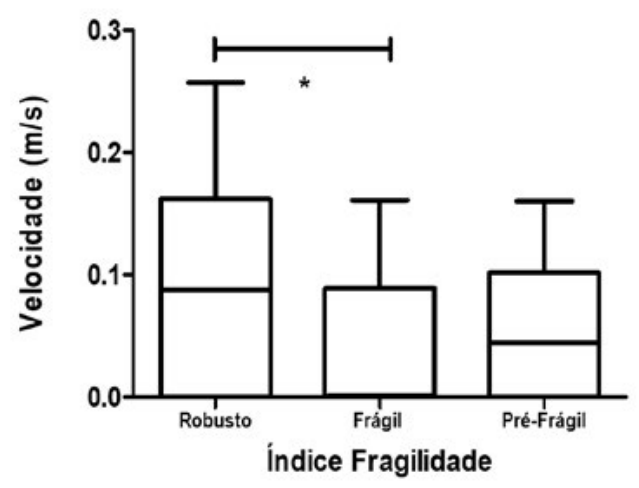

(B)

Figura 4. Percentis da população (5 a 95\%), de acordo com seus respectivos perfis de fragilidade e média de velocidades $(\mathrm{m} / \mathrm{s})$ na tarefa de atenção

\section{PERCENTIS DE VELOCIDADEDO ALCANCE} TAREFA DE ATENÇÃO

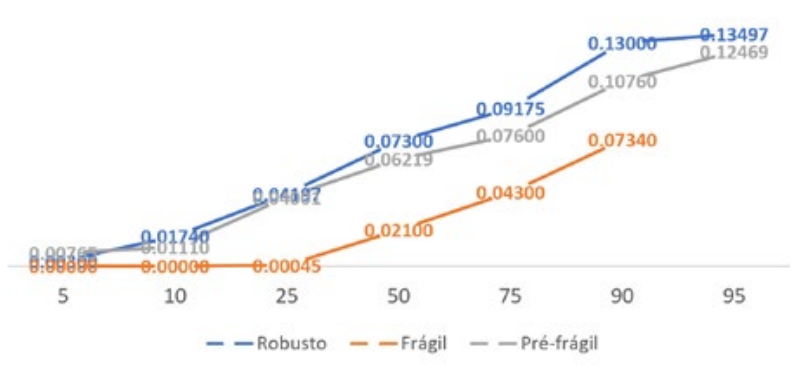

Figura 5. Percentis da população (5 a $95 \%$ ), de acordo com seus respectivos perfis de fragilidade e média de velocidades $(\mathrm{m} / \mathrm{s})$ na tarefa de memória

\section{PERCENTIS DE VELOCIDADEDO ALCANCE TAREFA DE MEMÓRIA}

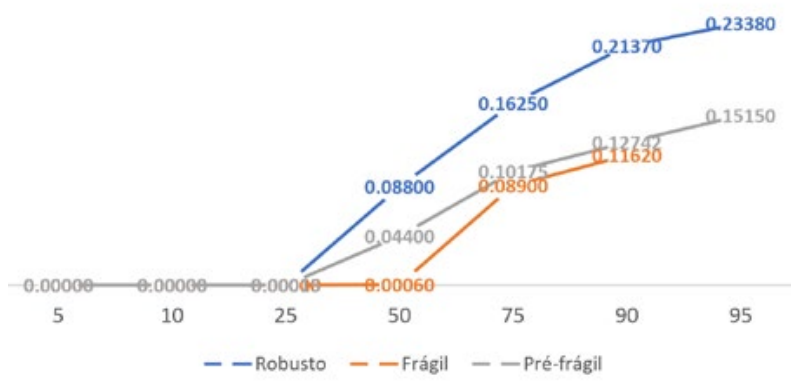


de alcance nas tarefas de memória demonstraram diferenças estatisticamente significativas entre o grupo Robusto e o grupo Frágil ( $\mathrm{p}=0,028)$. Indicadores baseados na função psicomotora, como é o caso do alcance durante tarefas de memória e atenção, têm recebido uma especial atenção e relevância na investigação atual, pois estudos recentes apontam que o declínio das Funções Executivas - tais como a atenção seletiva e a memória de trabalho - , podem ajudar e diferenciar idosos saudáveis de idosos com restrições funcionais. ${ }^{28-30}$

Diferentes testes funcionais que estão relacionados com o estado de fragilidade nos idosos têm sido implementados. A maioria dos artigos e revisões da literatura recomendam a medição da velocidade da marcha através do Timed up and Go (TUG), que é um teste de mobilidade de rápida aplicação e fácil reprodutibilidade. ${ }^{31}$ Para que este teste seja capaz de aferir dificuldades multidimensionais no idosos, incluindo na função executiva, alguns autores têm associado esse teste a tarefas secundárias (motoras e cognitivas), denominadas Timed Up and Goassociado com dupla tarefa (TUG-DT). A grande maioria destes pesquisadores utilizou a contagem regressiva, ${ }^{32}$ a nomeação de animais $^{33}$ e os cálculos matemáticos como estímulos cognitivos durante o teste. Assim, existe alguma diversidade de testes válidos para avaliar a fragilidade multidimensional para idosos ambulantes que são capazes de caminhar e de se locomover, mas existe uma lacuna importante de indicadores multidimensionais para idosos sem habilidade preservada de marcha.

A velocidade de alcance já tem sido investigada em estudos anteriores, como é o exemplo da investigação de Nima et al. ${ }^{13}$ Neste estudo, foi desenvolvido um método de avaliação para a extremidade superior e foram utilizados sensores de movimento para cálculo da velocidade de alcance durante a mesma tarefa realizada repetidamente. Neste estudo, foram incluídos 268 participantes com idade igual ou superior a 60 anos, que foram classificados como Robustos, Préfrágeis e Frágeis. $\mathrm{O}$ indicador de alcance obtido foi considerado válido como preditor de fragilidade física, mas sem capacidade de predizer fragilidade cognitiva. Uma das sugestões destes autores, dado os resultados inconclusivos para uma avaliação multidimensional do risco de fragilidade, foi a indicação, para estudos futuros, de inclusão de dupla-tarefa na medição da velocidade de alcance como complemento do método. Assim, o presente estudo é um contributo importante para a validação de um indicador de fragilidade baseado na função psicomotora dos idosos. Estes resultados podem ser de interesse para a prática clínica e facilitar a avaliação multidimensional da fragilidade no idoso, com especial atenção à restrição das funções executivas que são essenciais à preservação da autonomia funcional. ${ }^{34}$ Repare-se que, considerando os resultados obtidos nos idosos em estudo, parece existir uma tendência para intervalos de valores de velocidade de alcance na tarefa de atenção bastante diferentes entre a população Frágil (percentil 50-75\% - entre os $0,021 \mathrm{~m} / \mathrm{s}$ e $0,043 \mathrm{~m} / \mathrm{s}$ ) e a população Robusta (percentil da população de 50-75\% - entre 0,073m/s e 0,092m/s), comprovando-se a importância clínica da avaliação da velocidade de alcance. ${ }^{35}$

Aparentemente, pelos valores de percentis 50, idosos com velocidade de alcance inferiores a $0,073 \mathrm{~m} / \mathrm{s}$, nas tarefas de atenção, ou inferiores a $0,088 \mathrm{~m} / \mathrm{s}$, nas tarefas de memória, são indivíduos em risco de fragilidade. Esta conclusão e a sua importância para o diagnóstico clínico de fragilidade nos idosos merecem uma confirmação estatística de valores de corte em futuros estudos, através da análise ROC "Receiver Operating Characteristic". ${ }^{36}$

Apesar dos seus resultados promissores, foram identificadas diferentes limitações no presente estudo. Em primeiro lugar, o potencial da velocidade de alcance como indicador de fragilidade foi testado considerandose a comparação com um índice multidimensional baseado nos estudos de Jones et al. ${ }^{14}$

Noentanto, são vários os métodos de classificação da fragilidade validados cientificamente e que consideram diferentes domínios da saúde. Esta variabilidade entre metodologias de classificação de fragilidade prejudica a comparabilidade dos resultados neste contexto, em específico a validação de novos indicadores. Por outro lado, a validade preditora da velocidade de alcance nos testes AA e AM precisa de confirmação estatística em estudos prospectivos longitudinais e de recorrer à análise estatística avançada. Em terceiro lugar, para avaliar a especificidade do teste AM na identificação da fragilidade em idosos, futuros estudos devem implementá-lo em populações específicas com deficit cognitivo e de memória.

\section{Conclusão}

Para o processo de identificação da fragilidade em idosos, a escolha de um método simples e rápido, que implique poucos recursos e possibilite a avaliação 


\section{Artigo original}

multidimensional, é essencial para democratizar o processo de avaliação e possibilitar a sua interpretação por profissionais não especialistas. Por conseguinte, $\mathrm{o}$ teste de $\mathrm{AA}(<0,073 \mathrm{~m} / \mathrm{s})$ e $\mathrm{AM}(<0,088 \mathrm{~m} / \mathrm{s})$ demonstrou ser um indicador rápido, de fácil aplicação, de baixo custo e válido para identificar estados frágeis em idosos institucionalizados.

\section{Referências}

1. Nations U. World Population Prospects - The 2015 Revision Key Findings and Advance Tables. 2015

2. $\quad$ NE. Projeções da População Residente. 2017.

3. Fried LP, Tangen CM, Walston J, et al. Frailty in Older Adults: Evidence for a Phenotype. Journals Gerontol Ser A [internet] 2001 Mar 1;56(3):M146-57.

4. Rodríguez-Mañas $L$, Féart $C$, Mann $G$, et al. Searching for an operational definition of frailty: a Delphi method based consensus statement: the frailty operative definition-consensus conference project. J Gerontol A Biol Sci Med Sci [intemet]. 2012/04/16. 2013 Jan;68(1):62-7.

5. Walston J, Hadley EC, Ferrucci L, et al. Research Agenda for Frailty in Older Adults: Toward a Better Understanding of Physiology and Etiology: Summary from the American Geriatrics Society/National Institute on Aging Research Conference on Frailty in Older Adults. J Am Geriatr Soc [internet]. 2006 Jun $1 ; 54(6): 991-1001$

6. Gielen E, Verschueren S, O'Neill TW, et al. Musculoskeleta Frailty: A Geriatric Syndrome at the Core of Fracture Occurrence in Older Age. Calcif Tissue Int [Intemet]. 2012;91(3):16177.

7. Gavazzi GL. Frailty and ageing, concept and definitions. Rev Infirm. 2017;:66(236):18-20

8. Dent E, Kowal P, Hoogendijk EO. Frailty measurement in research and clinical practice: A review. Eur J Intem Med [internet]. 2016 Jun 1:31:3-10

9. Rockwood K, Howlett SE, MacKnight C, et al. Prevalence, Attributes, and Outcomes of Fitness and Frailty in Community-Dwelling Older Adults: Report From the Canadian Study of Health and Aging. Journals Gerontol Ser A [Internet]. 2004 Dec 1;59(12):1310-7.

10. Binotto MA, Lenardt MH, Rodríguez-Martínez M del C. Fragilidade física e velocidade da marcha em idosos da comunidade uma revisão sistemática . Vol. 52, Revista da Escola de Enfermagem da USP . scielo ; 2018.

11. Danilovich MKK, Diaz L, Corcos DM, et al. Relationship between SHARE-FI Frailty Scores and Physical Performance Measures in Older Adult Medicaid Recipients. Geriatr (Basel, Switzerland) [internet]. 2018 Aug 11:3(3):51

12. Ronthal M. Gait Disorders and Falls in the Elderly. Med Clin North Am [internet]. 2019;103(2):203-13

13. Toosizadeh N, Wendel C, Hsu C-H, et al. Frailty assessment in older adults using upper-extremity function: index development. BMC Geriatr [Internet]. 2017:17(1):117.

14. Jones D, Song X, Mitnitskj A, Rockwood K. Evaluation of a frailty index based on a comprehensive geriatric assessment in a population based study of elderly Canadians. Vol. 17, Aging clinical and experimental research. 2006, 465-471 p.
15. Yoo M, Kim S, Kim BS, et al. Moderate hearing loss is related with social frailty in a community-dwelling older adults: The Korean Frailty and Aging Cohort Study (KFACS). Arch Gerontol Geriatr [Internet]. 2019;83:126-30

16. Devore EE, Grodstein FSE. Sleep Duration in Relation to Cognitive Function among Older Adults: A Systematic Review of Observational Studies. Neuroepidemiology. 2016;46(1):57-78

17. Lee L, Patel T, Hillier $L M$, et al. Identifying frailty in primary care A systematic review. Geriatr Gerontol Int [intemet]. 2017 Oct 1:17(10):1358-77.

18. Malhotra R, Ang S, Allen JC, et al. Normative Values of Hand Grip Strength for Elderly Singaporeans Aged 60 to 89 Years: A Cross-Sectional Study. J Am Med Dir Assoc [internet]. 2016 Sep 1;17(9):864,e1-864,e7

19. Gor-García-Fogeda MD, Molina-Rueda F, Cuesta-Gómez A, et al. Scales to Assess Gross Motor Function in Stroke Patients: A Systematic Review. Arch Phys Med Rehabil [nternet]. 2014 Jun 1:95(6):1174-83

20. Folstein MF, Folstein SE, McHugh PR. "Mini-mental state": A practical method for grading the cognitive state of patients for the clinician. J Psychiatr Res [internet]. 1975;12(3):189-98

21. (GERM) N de E de G da SP de Ml. Avaliação Geríátrica. 2015

22. Sheikh Jl, Yesavage JA. Geriatric Depression Scale (GDS): Recent evidence and development of a shorter version. Clin Gerontol J Aging Ment Heal. 1986;5(1-2):165-73

23. Wade DT, Collin C. The Barthel ADL Index: A standard measure of physical disability? Int Disabil Stud [Internet]. 1988 Jan 1;10(2):64-7

24. Araújo F, Ribeiro JLP, Pinto C. Validação do Índice de Barthel numa amostra de idosos não institucionalizados. Rev Saúde Pública Port. 2007;25(2)

25. Lawton MP BE. Assessment of older people: self-maintain ing and instrumental activities of daily living. Gerontologist. 1969:9(3):179-86.

26. F.Araújo, Pais-Ribeiro J, Oliveira A, et al. Validação da Escala de Lawton e Brody numa amostra de idosos não institucionalizados. 2008

27. Rosa MDD, Pires M, Ferreira L, et al. Ssensory-motor upper extremity extremity function in institutionalised eldelry people role of emotional and cognitive status. In: Thomson Reuteurs Ageing Congress. 2019

28. Carlson MC, Xue Q- L, Zhou J, et al. Executive decline and dysfunction precedes declines in memory: the Women's Health and Aging Study II. J Gerontol A Biol Sci Med Sci [intemet]. 2009/01/31. 2009 Jan;64(1):110-7.

29. McGuinness B, Barrett SL, Craig D, et al. Passmore AP. Execu tive functioning in Alzheimer's disease and vascular dementia Int J Geriatr Psychiatry. 2010;25(6):562-8.

30. Kristensen CH. Funções executivas e envelhecimento - cognição e envelhecimento. Porto Alegre: Artmed; 2006. 91-111 p.

31. Mancilla S E, Valenzuela H J, Escobar C M. Rendimiento en las pruebas_Timed Up and Go_ y_Estación Unipodal_en adultos mayores chilenos entre 60 y 89 años. Vol. 143, Revista médica de Chile. scielocl; 2015. p. 39-46

32. Tang P-F, Yang H-J, Peng Y-C, et al. Motor dual-task Timed Up \& Go test better identifies prefrailty individuals than single-task Timed Up \& Go test. Geriatr Gerontol Int [Intemet]. 2015 Feb 1:15(2):204-10

33. Lima LCA, Ansai JH, Andrade LP, et al. The relationship between dual-task and cognitive performance among elderly par- 
Daniana Michelle e cols • A Velocidade do alcance durante tarefas de atenção e memória

ticipants who exercise regularly. Brazilian J Phys Ther [intemet]. 2015/04/27. 2015;19(2):159-66

34. Lezak MD, Howieson DB, Loring DW, et al. NoNeuropsychological assessment Title. 4th ed. Oxford University Press., editor. New York: American Psychological Association; 2004

35. Lindeboom J, Weinstein H. Neuropsychology of cognitive ageing, min- imal cognitive impairment, Alzheimer's disease, and vascular cognitive impairment. Eur J Pharmacol [internet]. 2004;490(1):83-6.

36. Aprahamian I, Cezar NO de C, lzbicki R, et al. Screening for Frailty With the FRAlL Scale: A Comparison With the Phenotype Criteria. J Am Med Dir Assoc [internet]. 2017 Jul $1 ; 18(7): 592-6$. 